\title{
A Revolutionary Material
}

\author{
Charles Radin
}

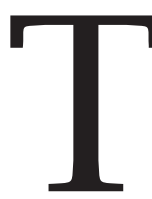

he 2011 Nobel Prize for Chemistry was awarded to Dan Shechtman for the discovery of quasicrystals, an exotic class of materials. The discovery was published in 1984 and was quickly treated as revolutionary, with front-page headlines in newspapers.

While the award was for chemistry, the revolution was more broadly based within the interdisciplinary subject of materials science. This can be described easily, and we will begin with a sketch of the idea. The multifaceted implications for mathematics are more complicated, and we will try to elucidate them afterwards.

The basic fact is that quasicrystals are equilibrium solids which are not crystalline. Not only is their pattern of atoms not crystalline, the pattern has a fascinating hierarchical structure. However, we emphasize that the hierarchical pattern is not essential to the revolutionary significance of quasicrystals to materials science.

It had been understood for many years, following the development of X-ray diffraction, that common inorganic solids (for instance all solids composed of only one chemical element) are crystalline, and great practical success followed from incorporating this into standard modeling, essentially by analyzing various perturbations of a crystalline atomic configuration. This is evident from basic textbooks on solid state physics from the 1970s. The startling fact uncovered by the discovery of quasicrystals was the existence of a previously unknown class of inorganic solids, of unknown

Charles Radin is professor of mathematics at the University of Texas, Austin. His email address is radin@math. utexas. edu.

This research was supported in part by NSF grant DMS1208941.

DOI: http://dx.doi.org/10.1090/noti954

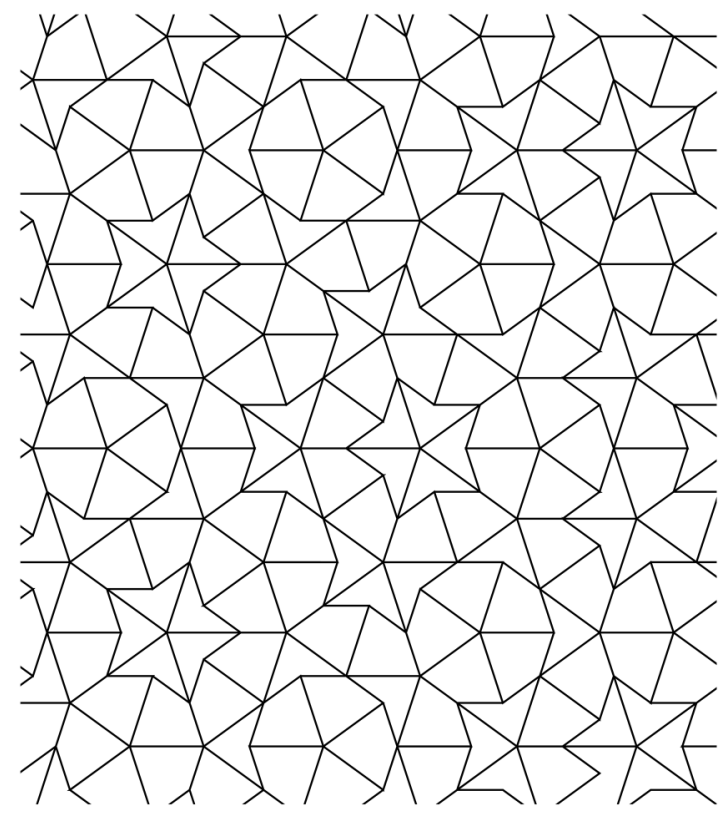

Figure 1. A Penrose kite and dart tiling of the plane.

diversity, for which a fundamentally different approach would be needed, specifically without the help of an underlying crystalline structure. That was the revolution in materials science.

As for the implications for mathematics, one path quickly developed from the hierarchical atomic patterns, which played a central role in the theory of Levine and Steinhardt based on aperiodic tilings such as the Penrose "kites and darts" (see Figure 1).

This led to interesting mathematics. The developments with which I am familiar are in the ergodic theory of aperiodic tilings and the theory of density in hyperbolic spaces, but there are 


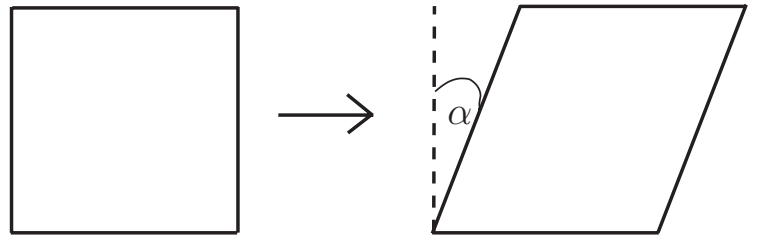

Figure 2. A strain angle $\alpha$.

undoubtedly many more developments inspired by the hierarchical structure.

That was the first, early line of development in mathematics coming from quasicrystals. But there is also significant mathematics intimately related to the revolutionary character of quasicrystals: the basic fact that quasicrystals are noncrystalline solids. Clarifying this mathematical connection is the goal for the rest of this article. This will require some review of the nature of solids and their modeling using equilibrium statistical mechanics, which we will motivate by focusing on a certain phenomenon.

To understand a material scientifically, one typically studies the experimental response to a disturbance; one kicks it and examines the reaction. Electrical conduction concerns the response to an applied voltage, sound propagation concerns the response to a (rapidly varying) applied pressure, elastic stress coefficients model the response to applied mechanical forces, and so on. Let us explore mechanical forces in more depth through the following specific problem.

It is natural that if you tried to stand on an ocean surface you would sink, because the water molecules would move out of the way of the force applied by your feet. Why then can you stand on a glacier? It turns out that, the more one analyzes these two contrasting material responses, of water and of ice, the more intriguing the question becomes, and we will use that idea to clarify the significance within mathematics of the discovery of quasicrystals. So we will keep in mind the problem:

\section{(1) Why can you stand on ice but not water?}

We begin with a certain classification of applied mechanical forces, or stresses. Suppose we have one balloon filled with water and another filled by a single block of ice. The possible stresses we could apply to the balloons are commonly classified into "pressure", which tends to change the volume but not the shape of the balloon, and "shear stress", which tends to change the shape but not the volume. Shape is quantified by angles denoting "strain"; see Figure 2.

We can view the force and the associated geometric change as responses to one another: applying a force yields a change in the geometry of the bulk material, and effecting a change in the geometry is resisted by a corresponding force from the material. The response is generally a nonlinear function of its cause, but the coefficient of the linear approximation is useful. The linear coefficient of response forces to changes in geometry are called moduli: (elastic) bulk modulus for pressure and (elastic) shear modulus for shear.

Getting back to our question (1) and the need to distinguish the response of ice from that of water, we choose to concentrate on shear, in particular, the shear moduli of ice and water. Pressure would be much simpler to analyze but of little value, since water is an incompressible fluid with almost the same bulk modulus as ice. But water deforms rather than supports any (static) shear at all, while ice is hard to deform, so the shear modulus of water is zero while that for ice is large. So, to answer (1) we shall try to understand through models why the shear moduli of water and ice are so different. Perhaps we can also reverse focus and ask whether this difference is the key to the fundamental difference between water and ice.

We will explore our problem now in more depth, beginning with the thermodynamic model of matter as an intermediate step towards the statistical mechanics model. For convenience we will restrict our modeling to "simple" materials which are (macroscopically) homogeneous, isotropic, uncharged, and of only one chemical species, and which are not acted on by magnetic, electric, or gravitational forces. The model will thus be restricted to questions of internal energy, such as the transfer of energy between two systems in contact, and the interaction of these with mechanical operations on the systems. A typical application might concern the energy of a gas in the chamber of a piston, the whole bathed in a fluid at fixed temperature, when the chamber of the piston is expanded.

The formalism of thermodynamics makes use of a quantity called the entropy density of the system. Experiment demonstrates that a simple system can be put into thermal equilibrium, where it has a range of well-defined equilibrium states, parameterized in several equivalent ways but, for instance, by the two quantities of energy density $e$ and mass density $m$, so that all thermodynamic quantities, including the entropy density and the various mechanical properties, have unique values for given $(e, m)$. Furthermore, it is found that all thermodynamic properties are computable from the entropy density function, $s(e, m)$. For instance, $\partial s / \partial e$ is inversely proportional to the temperature. A transform of $s(e, m)$, called the Gibbs free energy density, $g(P, T)$-basically a Legendre transform of $s(e, m)$ - can play a role alternative to $s(e, m)$ but with variables $P, T$, the pressure and temperature 
respectively.

(2) All thermodynamic properties are uniquely determined by the entropy density $s(e, m)$, or, alternatively, by the Gibbs free energy density, $g(P, T)$.

We next show how useful this observation can be in the modeling of mechanical properties.

The experimental states of bulk matter in thermal equilibrium can be organized into "phases". A phase is a set of states in an open connected subset of the parameter space $\{(P, T)\}$ which is maximal with respect to the property that, within that subset, all thermodynamic properties are analytic. From (2) it suffices to require this of just $g(P, T)$, so the boundary of a phase consists of singularities of $g(P, T)$.

The simplest phase of any material is the (isotropic) fluid phase, the phase which contains all $(P, T)$ with $P$ sufficiently low and $T$ sufficiently high. The complement of the fluid phase for any material contains those $(P, T)$ with $P$ sufficiently high and $T$ sufficiently low, and it contains one or more distinct "solid" phases, typically with distinct crystal structure. See Figure 3, which includes the curves of singularities of $g(P, T)$ bounding the fluid and solid phases. We note that a phase can bound itself; in fact, the part of the boundary of the fluid phase at which that phase bounds itself is where the gas and liquid forms of the fluid phase coexist; see Figure 3.

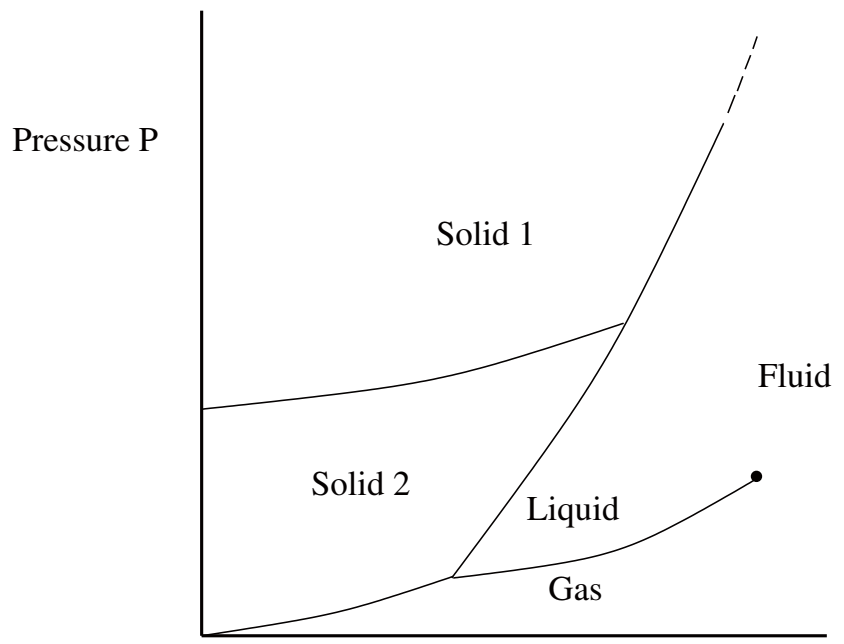

Temperature $\mathrm{T}$

Figure 3. A schematic experimental parameter space.

Using the language of phases, our problem is to understand why the shear moduli of the fluid and solid phases of water are so different. We sketched the thermodynamic analysis of simple matter, but this formalism does not address the causes of the phenomena it describes. Traditionally one goes to a deeper level of analysis, equilibrium statistical mechanics, for such understanding.

In brief, statistical mechanics tries to show how the thermodynamic properties of a material follow from the interaction of constituent particles, which we will call molecules; note that water and ice are both made from $\mathrm{H}_{2} \mathrm{O}$ molecules with the same interaction. The pressure of a gas, which has a natural meaning in terms of the mechanical properties of the bulk material, can also be understood in terms of momentum transfer between the molecules and between the molecules and the environment of the gas. The internal energy of the system can be understood in terms of the mechanical notions of potential and kinetic energies. However, the biggest step in the development of statistical mechanics, due to Boltzmann, was the model of entropy density as $(1 / V) \ln \left[\Gamma_{V}(e, m)\right]$, where $V$ is the volume of the material in space and $\Gamma_{V}(e, m)$ is the (high-dimensional) volume of the set of all joint states $c$ of the molecules that have total (kinetic plus potential) energy density $e(c)$ and mass density $m(c)$. The advantage of such modeling is that if we can compute the potential and kinetic energies between the molecules (even in a model with unrealistic interactions), we can, in principle, compute the entropy density $s(e, m)$ (or Gibbs free energy density $g(P, T)$ ), from which all thermodynamic information would follow. This would provide a deeper understanding of the thermodynamic properties; the all-important function $s(e, m)$, or $g(P, T)$, would follow from the interactions of the component molecules.

One feature of statistical mechanics that was omitted above, but which we need, concerns the phases. Since we are trying to understand the fundamental difference between these phases, it is necessary that we have access to them in our modeling. Now it has been known for many years that, if we accept the conventional meaning of phase boundaries as singularities of $s(e, m)$ or $g(P, T)$, we must take the limit of system size to infinity in the models. So to model the entropy density we use

$$
s(e, m)=\lim _{V \rightarrow \infty} \frac{1}{V} \ln \left[\Gamma_{V}(e, m)\right] .
$$

The above was a superficial introduction to the standard modeling of bulk materials in thermal equilibrium, including the notions of fluid and solid phases, but even though superficial, we can see that this modeling is insufficient to deal with our problem of the rigidity of solids. The difficulty is that the above theory does not address the response of a material to an applied shear 
strain! In fact, it has been proven that the above entropy density $s(e, m)$ and Gibbs free energy density $g(P, T)$ are independent of the shape of the material, and indeed we can look up material properties without specifying the shape of our material sample. And if $g(P, T)$ is independent of shape, how can we compute from it a response to changing the shape? This is our problem: for a macroscopic system of interacting molecules in thermal equilibrium, how do we model the response to shear strain and, in particular, show that it is high for state parameters $(P, T)$ corresponding to the solid phase but identically zero for $(P, T)$ corresponding to the fluid phase?

We will sketch two approaches to this matter. The first, by Aristoff and the author, gets around the above difficulty by three steps. One is the observation that the response to strain is in fact computable using the statistical mechanics of a finite system before taking the limit in system size. The next idea is a bit technical, namely, to use a response of a simpler nature than the reaction force of the system, i.e., the volume or mass density of the system. That is, one changes the shape and measures whether or not the volume changes. This is mildly counterintuitive, since shear is not supposed to change volume, but indeed it can, and this is actually a well-known phenomenon of sand called dilatancy. More formally, it is reasonable if we note that, because of the singularities of the free energy all along the boundary separating phases, $g(P, T)$ is intuitively a completely different function in different phases. So we might well expect every thermodynamic quantity, including the mass density, to be singular as $(P, T)$ crosses the boundary between phases.

The last idea is to look for a difference as we interchange limits, namely, the infinite size limit and the limit of infinitely small strain implicit in the derivative of mass density with respect to strain. More specifically, it is not hard to write a formula for a system of finite fixed size for the linear response, in other words, the derivative, of the (average) mass density with respect to shear strain. Then we can take the limit in the size of the system. To repeat: we take the limit of vanishingly small shear before we take the limit of infinite size. As noted above, we know that taking the limits in the other order cannot work, because as we take the infinite size limit, the free energy loses its dependence on the shape of the system. But does interchanging the limits help? The quantity we end up with, the volume limit of the derivative, is no longer the linear coefficient in an expansion of a response, since there is no response by the infinite system. But it still might be meaningful. We focused on the response because we thought it might distinguish water from ice, and the quantity we end up with may not be easy to interpret as a response, but it still could play a useful role in distinguishing fluid from solid in models. Does it do this, and if it does, what does it represent physically?

I said we can write a formula for the response, but it is a complicated integral, with parameters $P, T$, and $V$ in high dimension, and I did not say we could compute it analytically or even get useful qualitative information from it. The only evidence there is for the above theory comes from simulation in a standard model called "hard disks". For that model one can let a computer (actually many computers) apply Monte Carlo techniques to simulate the desired equilibrium quantities, and the result is that the linear response of mass density with respect to shear strain jumps from identically zero to nonzero precisely (within error!) as the thermodynamic parameter values cross the phase transition boundary between fluid and solid. So it seems to work precisely as desired in an important model, though this still leaves open its physical interpretation.

We now note a somewhat different approach to our basic problem (1) of distinguishing water from ice by Sausset, Biroli, and Kurchan, which uses the response to a time-dependent shear, a shear with constant strain rate, a standard quantity when analyzing fluids. The linear response of a fluid to a constant shear strain rate, namely, the (linear coefficient in the) response force to that deformation rate, is called viscosity. Sausset et al. analyze the viscosity of crystals using various traditional physics approximations and conclude that the difference between a fluid and a solid is that, within a solid the viscosity diverges in the limit of zero shear strain, while within a fluid the viscosity vanishes in the limit of zero shear strain. This offers a different intuitive picture of the essential difference between ice and water from that discussed earlier.

We cannot easily sketch the argument using viscosity, because it concentrates on time dependence, which is difficult to mesh with a well-defined notion of phase. In modeling requires time-independent equilibrium systems of infinite size. But we have included the approach specifically to bring up the important issue of time dependence, both in the physical material and in models of it. Throughout our discussion we have emphasized that quasicrystals are materials in thermal equilibrium, meaning they have the stability property that if perturbed by "annealing", the details of which are unimportant here, the system would return to its original state as measured by all thermodynamic properties. It is easy to prepare simple dilute gases in thermal equilibrium; all that is needed is to provide a steady environment of given pressure 
and temperature, and the system will naturally and quickly approach equilibrium. This is much harder to do with solids, and in practice many solids would change their state if annealed. Indeed, it is common to purposely prepare solids out of equilibrium in order to obtain desirable features: permanent magnets are examples, as are (structural) glasses such as window glass. From X-ray diffraction we know that the atomic positions in window glass are indistinguishable from that of the material in a liquid state at some $\left(P^{\prime}, T^{\prime}\right)$ corresponding to its manufacture process rather than being crystalline, as it would be in the true equilibrium state of the material at the $(P, T)$ of room pressure and temperature. And yet of course window glass is quite rigid. So a system which technically is just a very sluggish ("viscous") fluid, not in thermal equilibrium, can behave like an equilibrium solid. This makes (nonequilibrium) glasses notoriously difficult to model. When we model a quasicrystal we can use the fact that the material really is in thermal equilibrium, in effect that an infinite time limit has been taken; and clearly in our modeling we must analyze the proper order of taking that limit and the other limits of interest, namely, the (technically challenging) infinite size limit and the limit of zero strain. The proper simultaneous handling of these three limits is highly nontrivial, and such modeling issues cry out for the attention of serious applied mathematics. The traditional role for mathematics in open physics problems-for instance, concerning phase transitions-is to give proofs in standard, simplified models. However, because of the sophisticated technical issues involved, this problem seems to call for a different sort of role for mathematics, namely, in helping to determine the correct intuitive understanding of the phenomenon at hand: the difference between fluids and solids in thermal equilibrium.

In summary, there is a fundamental open problem in condensed matter physics to understand the essential difference between water and ice. In physics language we are looking for the right "order parameter" to distinguish the fluid and solid phases of matter in thermal equilibrium. It is perhaps surprising that no one has ever found an order parameter with which it could actually be proven, in some simple but convincing model, that a molecular system has a sharp transition between fluid and solid phases, so we could say that the shear modulus (or alternatively the derivative of density with respect to strain, or the viscosity, both sketched above) might play that role. Many of the older attempts to find such an order parameter focused on the difference in symmetry: the complete Euclidean symmetry of the fluid versus the crystalline symmetry of the solid. The existence of quasicrystals has affected this basic problem by showing that crystalline symmetry, and, by extension, perhaps symmetry itself, may not be relevant to an understanding of the fundamental difference between fluid and solid phases, and this fact motivated the attempts sketched above. The present physical theory of the wide range of phase transitions of materials developed, in part, by motivating significant progress in combinatorics and probability. Finally coming to grips with this most fundamental of phase transitions, the fluid/solid transition, may well require something different, a close collaboration of mathematics and physics in the basic modeling, and this is just one natural fallout of the quasicrystal revolution.

\section{Some References for the Physics}

These are the initial reports on the experimental discovery, and theory, of quasicrystals:

D. Shechtman, I. Blech, D. Gratias, and J. W. Cahn, Metallic phase with long-ranged orientational order and no translational symmetry, Phys. Rev. Lett. 53 (1984), 1951-1953.

D. Levine and P. J. Steinhardt, Quasicrystals: A new class of ordered structures, Phys. Rev. Lett. 53 (1984), 2477-2480.

Here are standard references on thermodynamics and statistical mechanics:

H. B. Callen, Thermodynamics, John Wiley, New York, 1960.

S.-K. Ma, Statistical Mechanics, World Scientific, Singapore, 1985.

Chapter 2 of the following contains an intriguing analysis of the role of "rigidity" in understanding phase transitions.

P. W. Anderson, Basic Notions of Condensed Matter Physics, Benjamin/Cummings, Menlo Park, 1984.

These are the two papers proposing theories of shear which are discussed in the article:

D. Aristoff and C. Radin, Rigidity in solids, J. Stat. Phys. 144 (2011), 1247-1255.

F. Sausset, G. Biroli, and J. Kurchan, Do solids flow? J. Stat. Phys. 140 (2010), 718-727.

\section{Some References for the Mathematical Formalism}

These are the standard references on the mathematical control of infinite size limits in statistical mechanics:

D. Ruelle, Statistical Mechanics; Rigorous Results, Benjamin, New York, 1969.

D. Ruelle, Thermodynamic Formalism, AddisonWesley, New York, 1978. 
Following are useful review articles on mathematical issues relevant to the article:

R. B. Griffiths, Rigorous results and theorems, in Phase Transitions and Critical Phenomena, vol. 1, C. Domb and M. S. Green, eds., Academic Press, New York, 1972, pp. 7-109.

O. E. Lanford, Entropy and equilibrium states in classical statistical mechanics, in Statistical Mechanics and Mathematical Problems, Battelle Rencontres, Seattle, 1971, Springer Lecture Notes in Physics, vol. 20, A. Lenard, ed., Springer-Verlag, Berlin-Heidelberg-New York, 1973, pp. 1-113.

J. L. Lebowitz, Statistical mechanics-A review of selected rigorous results, Annu. Rev. Phys. Chem. 19 (1968), 389-418.

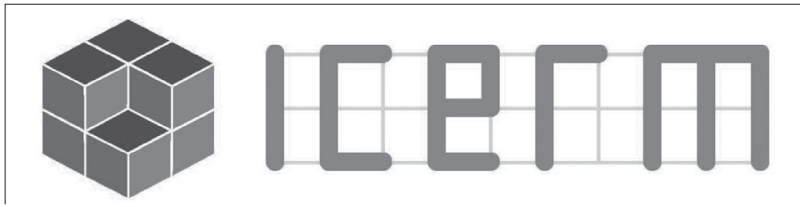

Institute for Computational and Experimental Research in Mathematics

\section{Low-dimensional Topology, Geometry, and Dynamics}

September 9 - December 6, 2013

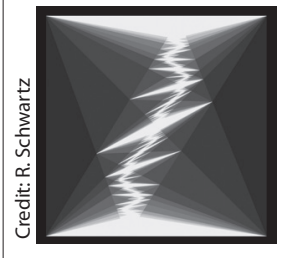

Program Description: This semesterlong program focuses on the recent impact of computation and experiment on the study of the pure mathematics sides of topology, geometry, and dynamics. Specific areas include 3-dimensional topology, the study of locally symmetric spaces, low-dimensional dynamics, and geometric group theory. Included are areas where computation has not yet had an impact, but might do so in the near future.

Workshop 1: Exotic Geometric Structures September 15-20, 2013

Workshop 2: Topology, Geometry and Group Theory, Informed by Experiment

October 21-25, 2013

Workshop 3: Geometric Structures in LowDimensional Dynamics

November 18-22, 2013

\section{Organizing Committee:}

Marc Culler, University of Illinois, Chicago

Nathan Dunfield, University of Illinois, Urbana-Champaign Walter Neumann, Barnard College, Columbia University Richard Schwartz, Brown University

Caroline Series, University of Warwick

Dylan Thurston, Barnard College, Columbia University Genevieve Walsh, Tufts University

Anton Zorich, IMJ, University Paris-7

To learn more about ICERM's programs, organizers,

confirmed program participants, and to submit an application, please visit our website:

\section{http://icerm.brown.edu}

Participation: ICERM welcomes applications for long- and short-term visitors. Support for local expenses may be provided. Full consideration will be given to applications received by March 15, 2013. Decisions about online applications are typically made 1-3 months before the program, as space and funding permit. ICERM encourages women and members of underrepresented minorities to apply.
About ICERM: The Institute for Computational and Experimental Research in Mathematics is a National Science Foundation Mathematics Institute at Brown University in Providence, Rhode Island. Its mission is to broaden the relationship between mathematics and computation.

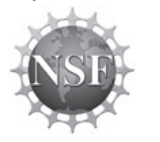

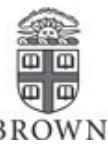

